\title{
Modeling Optimal Control of Cholera Disease Under the Interventions of Vaccination, Treatment and Education Awareness
}

\author{
Hellen Namawejje ${ }^{1}$, Emmanuel Obuya ${ }^{2}$, \& Livingstone S. Luboobi ${ }^{3}$ \\ ${ }^{1}$ Department of Statistical Methods and Actuarial Science, Makerere University, P.O.Box 7062, Kampala, Uganda \\ ${ }^{2}$ Department of Mathematics, University of Kisubi, P.O.Box 182, Entebbe, Uganda \\ ${ }^{3}$ Institute of Mathematical Sciences, Strathmore University P. O. Box 59857-00200, Nairobi, Kenya \\ Correspondence: Hellen Namawejje, School of Statistics and Planning, Makerere University, Kampala, Uganda. Tel: \\ 265-7750-70000.
}

Received: August 2, 2018 Accepted: August 20, 2018 Online Published: September 11, 2018

doi:10.5539/jmr.v10n5p137 URL: https://doi.org/10.5539/jmr.v10n5p137

\begin{abstract}
Cholera is virulent disease that affects both children and adults and can kill within hours. It has long been, and continues to be, a global threat to public health regardless of the advancement of medical science and health care service available. In this paper we formulate and analyze a mathematical model of the dynamics and optimal con- trol strategies for cholera epidemic. We present and analyze a cholera model with controls: $\mathrm{u} 1$ for vaccination of the human population, $\mathrm{u} 2$ for treatment and $\mathrm{u} 3$ for health education campaigns. The basic reproductive number R0; the effective reproductive number Re; as well as disease free equilibrium and endemic equilibrium points are derived. We establish the conditions for optimal control of the cholera disease using the Pontryagin's maximum principle and simulate the model for different control strategies. The results show that vaccination and education campaigns should be applied from start of the epidemic in any community faced with cholera disease.
\end{abstract}

Keywords: cholera epidemic, optimal control, Pontryagin's maximum principle, Basic and effective reproductive numbers

\section{Introduction}

Cholera is an acute, infectious illness caused by infection of the intestine with the bacterium referred to as Vibrio cholera (Vogt et al., 2010). The disease affects both children and adults. An estimated 3-5 million cases and over 100,000 deaths occur each year around the world.

Cholera was discovered in 1883 by the great German bacteriologist Robert Koch (1843-1910). As head of a commission investigating the disease, Koch went to Egypt where an epidemic was taking place and there he found some sort of bacterium in the intestines of those dead of cholera but could not differentiate between animals infected with the disease and those without.

Vogt et.al. (2010) also cited that Koch later in 1883 went to India, where he wrote that he succeeded in isolating "a little bent bacilli, like a comma'. He had then discovered that the bacteria thrived in damp dirty linen and moist earth and in the stools of patients with the disease.

Al-arydah et.al. (2014) noted that in recent years there have been a strong trend of cholera outbreaks in developing countries, including Haiti (2010 - 2011), Cameroon (2010 - 2011), Kenya (2010), Vietnam (2009), Zimbabwe (2008 2009), Iraq (2008), the Democratic Republic of Congo (2008), India (2007) among others.

In Uganda the most recent information on cholera suggest that between (4th - 10th January 2016) the ministry of health reported 32 cases in Hoima, 2 cases in Moroto and 2 cases in Nebbi districts. Hoima district reported two cholera related deaths, amounting to $6.3 \%$. Almost half of the people infected with Cholera were children. Younger children are reportedly exposed to Cholera during recreation activities such as swimming in water bodies where the health conditions are below standard levels (Bashiru and Asokan, 2016).

Ali et.al. (2012) cited out that the cholera bacterium is usually found in water or food sources that have been contaminated by feaces from a person infected with cholera. Cholera is most likely to be found and spread in places with inadequate water treatment, poor sanitation, and inadequate hygiene. One can get cholera by drinking water or eating food contaminated with the cholera bacterium. In an epidemic, the source of the contamination is usually the feaces of an infected person that contaminates water or food. The disease can spread rapidly in areas with inadequate treatment of sewage and drinking water. The disease is not transmitted through contact in most cases (Barzley et.al., 2013). 
Gleick and Ajami, (2014) cited out that the symptoms of cholera are not easily noticed but can be severe and the main features of the disease are; profuse watery diarrhea, vomiting, and leg cramps. In these people, rapid loss of body fluids leads to dehydration and shock. Without treatment, death can occur within hours. Symptoms typically appear in 2-3 days or even in hours. Also Wang and Modnak (2011) modeled cholera dynamics with controls were they applied the optimal control theory to seek the cost-effective solution of multiple time-dependent intervention strategies against cholera outbreaks.

Further more different mathematical models have been developed to predict how best cholera can be controlled by various researchers, for example, Devipriya and Kalaivani, (2012) worked on the optimal control of multiple transmission of water-borne diseases; Isere et al. (2014) formulated a model for Optimal control of outbreaks of cholera in Nigeria. Miller (2009) formulated a general optimal control system applied to population and disease models. Chao (2011) also carried out research on mathematical modeling of cholera dynamics basically based in Bangladesh and Haiti. He used the analytic approach by applying the observed cholera outbreak values on the basic SIR model for modeling the infection of diseases.

Neilan et al. (2010) in their work, they incorporated essential components such as hyper infections, short-lived bacterial state, a separate class for mild human infections, and waning disease immunity. They also applied optimal control theory, did numerical simulations and parameter sensitivity analysis to investigate the best combination of different cost-effective strategies with multiple intervention while Zhou and Cui (2011) investigated stability analysis of a cholera model with vaccination. In this paper, we improve the work done by Zhou and Cui (2011) to understand the optimal control of cholera disease under the interventions of vaccination, treatment, and awareness education as our control parameters. By applying the optimal control theory we want to derive the best optimal solution to mitigate the risk of cholera spread in our communities.

\section{Model Formulation}

We consider an SVIRB model, where $S(t)$ denotes the number of susceptible individuals, $V(t)$ denotes the number of vaccinated individuals, $I(t)$ denotes the number of infected individuals, $R(t)$ denotes the number of recovered individuals and $B(t)$ denotes the pathogen environment at time t. We include three control interventions, that is: vaccination, treatment and education awareness. Some of the individuals are vaccinated while others are not.

A proportion of the unvaccinated join the susceptible group while the remaining fraction joins the vaccinated class. Some of the individuals that are exposed and acquire the disease are treated and at recovery they join class $R(t)$. Vaccination does not give permanent immunity to those that are vaccinated.

In this model, we assume a recruitment rate $A$ into $S(t)$ through birth or immigration. Individuals at $S(t)$ can get infected either through contact with the infected individuals or through contact with the infected environment. Let $\beta$ be the rate of contact that is sufficient for transmission of the disease between classes. The infected individuals $I(t)$ join the recovery class $R(t)$ at a rate $\alpha$ and die due to the disease at a rate $d$. Due to the nature of the disease, individuals can lose immunity and become susceptible again at a rate $b$. A proportion $\rho$ of the susceptible population is vaccinated. All the classes experience a natural death at a rate $\mu$. We further note that, the pathogen infected population is generated at a rate $\gamma$ while the contribution rate of infected individuals to the pathogen growth in environment is $\eta$ and the cholera virus die naturally at a rate $\mu_{1}$. Table 1 shows the description of the variables used in the model.

Table 1. State Variables of the Model

\begin{tabular}{l|l}
\hline Variables & Description \\
\hline$S(t)$ & Number of susceptible at time $\mathrm{t}$ \\
$V(t)$ & Number of vaccinated people at time $\mathrm{t}$ \\
$I(t)$ & Number of infectious at time $\mathrm{t}$ \\
$R(t)$ & Number of recovered children time $\mathrm{t}$ \\
$B(t)$ & The pathogen population in the contaminated environment at time $\mathrm{t}$ \\
\hline
\end{tabular}




\subsection{Model Assumptions}

The following are the assumptions made when formulating the model:

(a) Vaccination, treatment and education are implemented continuously.

(b) Susceptible individuals are vaccinated at a rate of $u_{1}$ so that $u_{1} S(t)$ individuals are added to the vaccinated class

(c) All infected individuals are treated at a rate of $u_{2}$ so that $u_{2} I(t)$ individuals move to the recovered class.

(d) Educating the population about the disease reduces the degree of infection from the environment at a rate of $u_{3}$

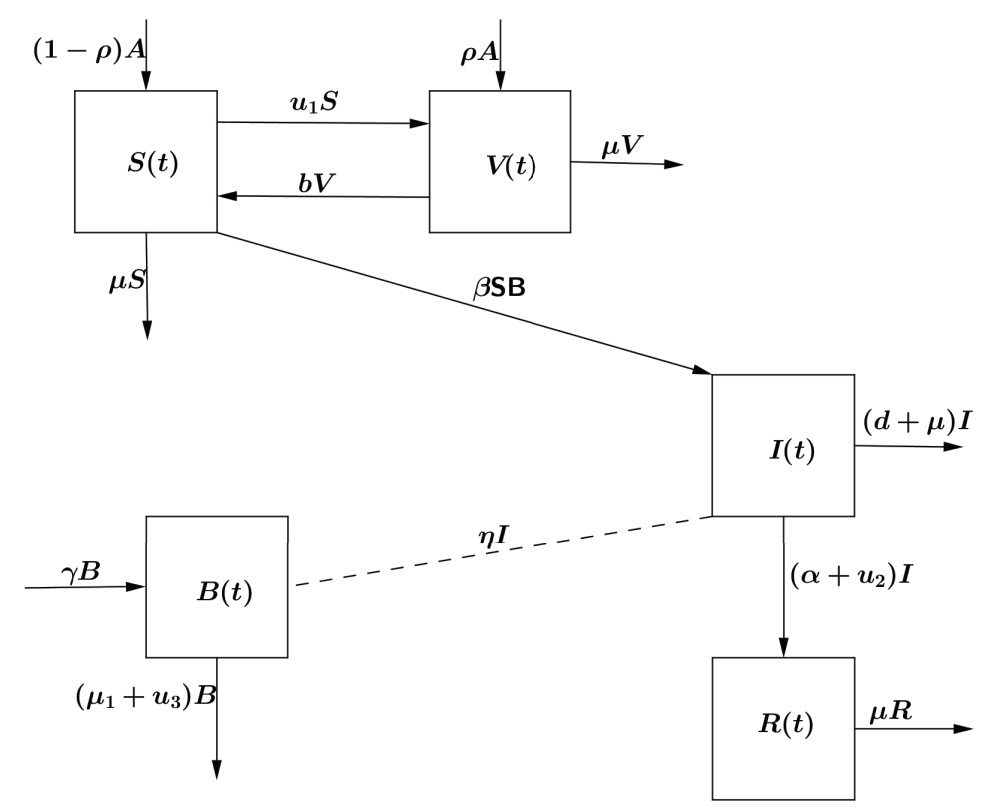

Figure 1. Dynamics of cholera model with vaccination, treatment and health education campaigns as controls

The dynamics of cholera as described above is summarized in the model flow diagram in Figure 1. The parameters are summarized in Table 2 .

Table 2. Parameters of the Model

\begin{tabular}{l|l}
\hline Parameters & Description \\
\hline$\rho$ & The fraction of the susceptible recruited individuals to be vaccinated \\
\hline$\alpha$ & The constant recovery rate \\
\hline$\beta$ & The rate of contact that is sufficient to transmit the disease \\
\hline$b$ & The rate at which the vaccine wears offs \\
\hline$\eta$ & The death rate due to cholera disease \\
\hline$\gamma$ & The rate at which infected contribute to the concentration of vibrios \\
\hline$\mu_{1}$ & The rate at which the pathogen population is generated \\
\hline$u_{1}$ & The rate of natural death. \\
\hline$u_{2}$ & The natural death rate of the cholera disease \\
\hline$u_{3}$ & The rate at which the susceptible population is vaccinated \\
\hline$A$ & The rate at which infected people are treated \\
\hline
\end{tabular}

In this model, we derive the optimal control strategy such that the total infection burden is reduced to minimum cost within a period of 100 days. From the description of the dynamics of cholera as depicted in Figure 1, we have the following set of ordinary differential equations system. 


$$
\begin{aligned}
\frac{d S}{d t} & =(1-\rho) A-\beta S(t) B(t)+b V(t)-u_{1} S(t)-\mu S(t), \\
\frac{d V}{d t} & =\rho A+u_{1} S(t)-b V(t)-\mu V(t), \\
\frac{d I}{d t} & =\beta S(t) B(t)-(d+\alpha+\mu) I(t)-u_{2} I(t), \\
\frac{d R}{d t} & =\alpha I(t)-\mu R(t)+u_{2} I(t), \\
\frac{d B}{d t} & =\gamma B(t)+\eta I(t)-\mu_{1} B(t)-u_{3} B(t)
\end{aligned}
$$

with initial conditions

$S(0)=S_{0}, \quad V(0)=V_{0}, \quad I(0)=I_{0}, \quad R(0)=R_{0}, B(0)=B_{0}$.

\subsection{Model Analysis}

Under this Subsection, we start by first establishing the existence of an invariant region and showing that all solutions of the model (1)-(5) are non-negative and bounded for all $t \geq 0$.

\subsubsection{Invariant Region}

The solutions of equation (1)-(5) are positive for all $t>0$ if they enter the invariant region $\Omega$

\section{Proof}

Let $\mathbf{\Omega}=(S, V, I, R, B) \in \mathbf{R}_{+}^{\mathbf{5}}$ be the solutions of the model with non-negative initial conditions. From the interactions the total population is given by:

$$
N_{H}=S+V+I+R
$$

That is:

Adding the equations (1)-(5), we obtain

$$
\frac{d N_{H}}{d t}=\frac{d S}{d t}+\frac{d V}{d t}+\frac{d I}{d t}+\frac{d R}{d t}
$$

$$
\frac{d N_{H}}{d t}=A-\mu N-d I
$$

In the absence of the disease $d=0$ and (6) becomes

$$
\frac{d N_{H}}{d t} \leq A-\mu N
$$

We integrate (7), with $e^{\mu t}$ as the integrating factor and we obtain

$$
N_{H} e^{\mu t} \leq \frac{A}{\mu} e^{\mu t}+C
$$

$\mathrm{C}$ is a constant of integration. Applying initial conditions, when $t=0$ and $N(0)=N_{0}$, this gives

$$
N_{H} \leq \frac{A}{\mu}+\left(N_{0}-\frac{A}{\mu}\right) e^{-\mu t}
$$

For $N_{H}(0)<\frac{A}{\mu}$, we have from (8)

$$
0 \leq N_{H}(t) \leq \frac{A}{\mu}, \text { for all } t>0
$$

This implies that $N_{H}(t)$ is bounded above. That is,

$$
N_{H}(t) \leq N_{H}^{*}=\max \left\{N_{0}, \frac{A}{\mu}\right\} .
$$

Similarly, considering (5), we let the total population of the pathogen be $B(t)$, we have

$$
\begin{aligned}
& \frac{d B(t)}{d t}=\gamma B(t)+\eta I(t)-\mu_{1} B(t)-u_{3} B(t) \\
& \frac{d B(t)}{d t}=\gamma B(t)+\eta I(t)-\mu_{1} B(t)-u_{3} B(t) \leq\left(\gamma-\mu_{1}-u_{3}\right) B(t)+\eta N_{H} \\
& \frac{d B(t)}{d t}=\leq\left(\gamma-\mu_{1}-u_{3}\right) B(t)+\eta N_{H}
\end{aligned}
$$


From (10) we have $N_{H}(t) \leq N_{H}^{*}$ which implies that

$$
\frac{d B(t)}{d t}=\leq\left(\gamma-\mu_{1}-u_{3}\right) B(t)+\eta N_{H} \leq\left(\gamma-\mu_{1}-u_{3}\right) B(t)+\eta N_{H}^{*}
$$

Integrating both sides of (12), we have

$$
B(t) \leq \frac{\eta N_{H}^{*}}{\left(-\gamma+\mu_{1}+u_{3}\right)}\left[1-e^{-\left(-\gamma+\mu_{1}+u_{3}\right) t}\right]
$$

Thus the pathogen population size $B(t) \leq \frac{\eta N_{H}^{*}}{\left(-\gamma+\mu_{1}+u_{3}\right)}$ provided $\left(\mu_{1}+u_{3}\right)>\gamma$. Therefore the feasible solution set of the pathogen population of the system (1)-(5) enters the region

$$
\left\{\mathbf{\Omega}_{\mathbf{B}}=B(t) \in \mathbf{R}_{+}^{\mathbf{1}} \mid B(t) \geq 0 \frac{\eta N_{H}^{*}}{\left(-\gamma+\mu_{1}+u_{3}\right)}\right\} .
$$

Thus the invariant region of the system (1)-(5) is given by

$$
\begin{aligned}
& \left\{\boldsymbol{\Omega}=(S, V, I, R, B) \in \mathbf{R}_{+}^{5} \mid(S, V, I, R, B) \geq 0 ;\right. \\
& \left.N_{H}(t) \leq N_{H}^{*} ; \quad B(t) \leq \frac{\eta N_{H}^{*}}{\left(\mu_{1}+u_{3}-\gamma\right)}\right\} .
\end{aligned}
$$

where $N_{H}(t)$ is the total population of individuals and $\mathrm{B}(\mathrm{t})$ is the pathogen population. Thus every solution with initial condition in $\Omega$ remains in $\Omega$ for all $t \geq 0$. The proposed model with equations (1)-(5) is biologically feasible and positively invariant.

\subsubsection{Positivity of Solutions}

Let the initial data be

$$
\{(S(0), V(0), I(0), R(0), B(0)) \geq 0\} \in \mathbf{R}_{+}^{5} .
$$

Then, the solution set

$$
\{S(t), V(t), I(t), R(t), B(t)\}
$$

of the system (1)-(5) is non-negative for all $t>0$.

\section{Proof:}

From (1)

$$
\begin{aligned}
\frac{d S}{d t} & =(1-\rho) A-\beta S(t) B(t)+b V(t)-u_{1} S(t)-\mu S(t), \\
\frac{d S(t)}{d t} & \geq-\left(\beta B(t)+u_{1}+\mu\right) S
\end{aligned}
$$

By integrating on both sides with respect to $\tau$ for $\tau=0$ to $\tau=t$ and applying initial conditions, we have,

$$
S(t) \geq S(0) e^{\int_{\tau=0}^{\tau=t}-\left(\beta(\tau)+u_{1}+\mu\right) d \tau} \geq 0
$$

Considering (2)

$$
\frac{d V}{d t}=\rho A+u_{1} S(t)-b V(t)-\mu V(t) \geq-(b+\mu) V(t)
$$

Integrating on both sides with initial conditions, we get,

$$
V(t)=V(0) e^{-(b+\mu) t} \geq 0
$$

Similarly from the other equations (3), (4) and (5) respectively are derived and we have the following results:

$$
\begin{aligned}
& I(t) \geq I(0) e^{-\left(d+\alpha+\mu+u_{2}\right) d t} \geq 0 \\
& R(t) \geq R(0) e^{-\mu t} \geq 0 \\
& B(t) \geq B(0) e^{\int_{\tau=0}^{\tau=t}\left(-\gamma+u_{3}+\mu_{1}\right) d \tau} \geq 0 .
\end{aligned}
$$




\subsubsection{Disease Free Equilibrium (DFE)}

Let the disease free equilibrium (DFE) point be denoted by $X_{0}=\left(S^{0}, V^{0}, 0,0,0\right)$. The disease free equilibrium point is obtained by equating the right hand of system (1)-(5) to zero.

$$
\begin{aligned}
& (1-\rho) A-\beta S(t) B(t)+b V(t)-u_{1} S(t)-\mu S(t)=0, \\
& \rho A+u_{1} S(t)-b V(t)-\mu V(t)=0, \\
& \beta S(t) B(t)-(d+\alpha+\mu) I(t)-u_{2} I(t)=0, \\
& \alpha I(t)-\mu R(t)+u_{2} I(t)=0, \\
& \gamma B(t)+\eta I(t)-\mu_{1} B(t)-u_{3} B(t)=0,
\end{aligned}
$$

In the absence of the disease, $(I, R, B)=0$. Therefore the disease free equilibrium is given as

$$
X_{0}=\left\{\frac{A}{\mu}\left(\frac{b+(1-\rho) \mu}{\left(u_{1}+b+\mu\right)}\right), \frac{A\left(u_{1}+\rho \mu\right)}{\mu\left(u_{1}+b+\mu\right)}, 0,0,0\right\}
$$

\subsection{Reproduction Number, $R_{0}$}

Under this Subsection, we derive the expression for the basic reproduction number of the model (1)-(5) using the next generation method as by Van den Driessche and Watmough (2002). The basic reproduction number is one of the most useful threshold parameters that characterizes mathematical problems concerning infectious diseases. This parameter is useful because it helps determine whether an infectious disease will spread through a population or not. We consider

(i) $\mathcal{F}_{i}(x)$ be the rate of appearance of new infections in compartment i.

(ii) $\mathcal{V}_{i}^{+}(x)$ be the rate of transfer of individuals into compartment i by all other means, other than the epidemic.

(iii) $\mathcal{V}_{i}^{-}(x)$ be the transfer of individuals out of the compartment $\mathrm{i}$.

The disease transmission model consists of the system of equations

$$
x_{i}^{\prime}=f_{i}(x)=\mathcal{F}_{i}(x)-\mathcal{V}_{i}(x)
$$

where

$$
\mathcal{V}_{i}=\mathcal{V}_{i}^{-}(x)-\mathcal{V}_{i}^{+} .
$$

From the disease-free equilibrium point $X_{0}$, we then compute matrices $\mathrm{F}$ and $\mathrm{V}$ which are $m \times m$ matrices defined by

$$
F=\left[\frac{\partial \mathcal{F}_{i}}{\partial x_{j}}\left(S_{0}\right)\right]
$$

and

$$
V=\left[\frac{\partial \mathcal{V}_{i}}{\partial x_{j}}\left(S_{0}\right)\right] \text { with } 1 \leq i, j \leq m,
$$

where $m$ represents the infected classes and $\mathrm{F}$ is nonnegative, $\mathrm{V}$ is a nonsingular $\mathrm{M}$-matrix, $V^{-1}$ is nonnegative and also $F V^{-1}$ is non-negative (Van den Driessche and Watmough, 2002).

We then compute matrix $F V^{-1}$ referred to as the next generation matrix (Diekmann et al., 1990). The effective reproductive number $R_{e}$ is then defined as

$$
R_{e}=\rho\left(F V^{-1}\right)
$$

where $\rho(A)$ is the spectral radius of matrix A (or the maximum real part of the eigenvalues of A).

We now derive $\mathcal{F}_{i}$ and $\mathcal{V}_{i}$ of our model system (1)-(5) as:

$$
\mathcal{F}_{i}=\left[\begin{array}{c}
\beta S B \\
0 \\
0 \\
0 \\
0
\end{array}\right]
$$


and

$$
\mathcal{V}_{i}=\left[\begin{array}{c}
\left(d+\alpha+\mu+u_{2}\right) I \\
\left(\mu_{1}+u_{3}-\gamma\right) B-\eta I \\
-(1-\rho) A+\beta S B-b V+u_{1} S+\mu S \\
-\rho A-u_{1} S+(b+\mu) V \\
\mu R-\left(u_{2}+\alpha\right) I
\end{array}\right]
$$

At equilibrium point, $\mathrm{B}=\mathrm{I}=0$ and the initial conditions are assumed to be positive. Evaluating the equation (14) and (15) we obtain;

$$
F=\left[\begin{array}{cc}
0 & \beta S^{0} \\
0 & 0
\end{array}\right]
$$

Similarly, the Jacobian matrix of (15) is found and gives;

$$
V=\left[\begin{array}{cr}
\left(d+\alpha+\mu+u_{2}\right) & 0 \\
-\eta & \left(\mu_{1}+u_{3}-\gamma\right)
\end{array}\right]
$$

We then find the inverse of (17) which is used to compute $F V^{-1}$ and we get;

$$
F V^{-1}=\left[\begin{array}{cc}
\frac{\eta \beta S^{0}}{\left(\mu_{1}+u_{3}-\gamma\right)\left(d+\alpha+\mu+u_{2}\right)} & \frac{\beta S^{0}}{\left(\mu_{1}+u_{3}-\gamma\right)} \\
0 & 0
\end{array}\right]
$$

Therefore the effective reproductive ratio $R_{e}$ is obtained by taking the spectral radius (dominant eigenvalue) of the matrix $F V^{-1}$ is given by

Substituting the equivalent of $S^{0}$ we have;

$$
R_{e}=\frac{\eta \beta S^{0}}{\left(\mu_{1}+u_{3}-\gamma\right)\left(d+\alpha+\mu+u_{2}\right)}
$$

$$
R_{e}=\frac{\eta \beta A(b+(1-\rho) \mu)}{\mu\left(\mu_{1}+u_{3}-\gamma\right)\left(d+\alpha+\mu+u_{2}\right)\left(b+u_{1}+\mu\right)}
$$

The basic reproductive ratio $R_{0}$ is obtained from the effective reproductive number $R_{e}$ computed when all the control parameters are equal to zero

$$
R_{0}=\frac{\eta \beta A(b+(1-\rho) \mu)}{\mu\left(\mu_{1}-\gamma\right)(d+\alpha+\mu)(b+\mu)}
$$

\subsubsection{Endemic Equilibrium Point (EEP)}

According to Ugwa et.al. (2013) an endemic point is the state where the disease cannot totally be eradicated but remains in the population. For the disease to persist in the population, the immunized class, the susceptible class, the infected class, the infectious class and the recovered class must not be zero at equilibrium state. Let the endemic point be denoted by $E^{*}=\left(S^{*}, V^{*}, I^{*}, R^{*}, B^{*}\right)$ and also let the right hand side of model system (1)-(5) be equal to zero. Computing algebraically the EEP, $E^{*}$ is given as:

$$
\begin{aligned}
S^{*} & =\frac{A(b+(1-\rho) \mu)}{\mu R_{e}\left(b+u_{1}+\mu\right)} \\
V^{*} & =\frac{A}{\mu}\left(\frac{\rho A\left(b+u_{1}+\mu\right)+u_{1}(b+(1-\rho) \mu)}{R_{e}(b+\mu)\left(b+u_{1}+\mu\right)}\right) \\
I^{*} & =\frac{\beta(A(b+(1-\rho) \mu))\left((1-\rho) A+b\left(A \rho A R_{e}\left(b+u_{1}+\mu\right)+u_{1}(b+(1-\rho) \mu)\right)\right.}{\Phi}+ \\
& \frac{\left(\mu+u_{1}\right) A(b+(1-\rho) \mu)\left(\mu R_{e}\left(b+u_{1}+\mu\right)\right)}{\Phi} \\
R^{*} & =\frac{\beta(A(b+(1-\rho) \mu))^{2}\left((1-\rho) A+b\left(A \rho A R_{e}\left(b+u_{1}+\mu\right)+u_{1}(b+(1-\rho) \mu)\right)\right)}{\Delta}- \\
& \frac{\left(\mu+u_{1}\right) A(b+(1-\rho) \mu)\left(\mu R_{e}\left(b+u_{1}+\mu\right)\left(\rho+u_{2}\right)\right)}{\Delta} \\
B^{*} & =\frac{(1-\rho) A+b\left(A \rho A R_{e}\left(b+u_{1}+\mu\right)+u_{1}(b+(1-\rho) \mu)\right)}{\Psi}+ \\
& \frac{\left(\mu+u_{1}\right) A(b+(1-\rho) \mu)\left(\mu R_{e}\left(b+u_{1}+\mu\right)\right)}{\Psi}
\end{aligned}
$$


where

$$
\begin{aligned}
& \Phi=\left(d+\alpha+\mu+u_{2}\right)\left(\mu R_{e}\left(b+u_{1}+\mu\right)\right)\left(\beta A(b+\mu)\left(b+u_{1}+\mu\right)(b+(1-\rho) \mu)\right) \\
& \Delta=\left(\mu R_{e}\left(b+u_{1}+\mu\right)\right)\left(d+\alpha+\mu+u_{2}\right) \beta A\left(\mu R_{e}(b+\mu)\left(b+u_{1}+\mu\right)(b+(1-\rho) \mu)\right) \\
& \Psi=\beta A\left(\mu R_{e}(b+\mu)\left(b+u_{1}+\mu\right)(b+(1-\rho) \mu)\right)
\end{aligned}
$$

\section{Optimal Control Analysis}

In this Section we use the optimal control theory to investigate the behavior of the model system (1)-(5). Our main goal is to vaccinate as many susceptibles as to minimize the number of infected individuals due to cholera and the cost of this strategy. We fix the terminal time $T$, the problem is to minimize the objective functional $J$ given as:

$$
\mathcal{J}\left(u_{1}, u_{2}, u_{3}, u_{4}\right) \quad=\quad \min _{\left(u_{1}, u_{2}, u_{3}, u_{4}\right)} \int_{0}^{T}\left\{L_{1} S(t)+L_{2} I(t)+\frac{1}{2}\left(M_{1} u_{1}^{2}+M_{2} u_{2}^{2}+M_{3} u_{3}^{2}\right)\right\} d t
$$

where $L_{1}$ and $L_{2}$ are the weight constants of the susceptible and the infected groups respectively, whereas $M_{1}, M_{2}$ and $M_{3}$ are the constant relative cost weight parameters for vaccination, treatment and education campaigns efforts respectively which regulate the optimal control. We further assume that due to uncontrollable reasons, the cost of vaccination, treatment and education campaigns is nonlinear and quadratic as seen in the cost function (19). $M_{1} u_{1}^{2}$ represents the cost of vaccination, $M_{2} u_{2}^{2}$ represents the cost of treatment and $M_{3} u_{3}^{2}$ represents the cost of the education awareness.

Thus the Lagrange for the optimal control problem (19) is given by:

$$
\mathcal{L}\left(N_{H}, N_{B}, u_{i}\right)=\left\{L_{1} S(t)+L_{2} I(t)+\frac{1}{2}\left(M_{1} u_{1}^{2}+M_{2} u_{2}^{2}+M_{3} u_{3}^{2}\right)\right\}
$$

where $N_{H}$ is the total population of people, $N_{B}$ is the free pathogen environment and $u_{i}$ representing controls with $i=1,2,3$ as defined in Table 2. The Hamiltonian function $\mathrm{H}$ for our control problem is:

$$
\begin{aligned}
H= & L_{1} S(t)+L_{2} I(t)+\frac{1}{2}\left(M_{1} u_{1}^{2}+M_{2} u_{2}^{2}+M_{3} u_{3}^{2}\right)+ \\
& \lambda_{S}(t)\left((1-\rho) A-\beta S(t) B(t)+b V(t)-u_{1} S(t)-\mu S(t)\right)+ \\
& \lambda_{V}(t)\left(\rho A+u_{1} S(t)-b V(t)-\mu V(t)\right)+ \\
& \lambda_{I}(t)\left(\beta S(t) B(t)-(d+\alpha+\mu) I(t)-u_{2} I(t)\right)+ \\
& \lambda_{R}(t)\left(\alpha I(t)-\mu R(t)+u_{2} I(t)\right)+ \\
& \lambda_{B}(t)\left(\gamma B(t)+\eta I(t)-\mu_{1} B(t)-u_{3} B(t)\right)
\end{aligned}
$$

We therefore need to numerically solve our optimal control functions $u_{1}^{*}, u_{2}^{*}$, and $u_{3}^{*}$ that satisfy our optimal control condition:

$$
\left.J\left(u_{1}^{*}, u_{2}^{*}, u_{3}^{*}\right)=\min \left\{J\left(u_{1}, u_{2}, u_{3}\right):\left(u_{1}, u_{2}, u_{3}\right) \in \mathbf{U}\right)\right\}
$$

where $\mathbf{U}$ is the set of admissible controls defined by

$$
\left\{\left(u_{1}, u_{2}, u_{3}\right): 0 \leq u_{1}, u_{2}, u_{3} \leq 1, t \in[0, T], u_{1}, u_{2} \text {, and } u_{3} \text { are lebesgue measurable }\right\}
$$

\subsection{The Optimal Control Solution}

Here we apply the Pontryagins maximum principle (PMP), (Pontryagin et.al., 1962). We use this principle to identify the adjoint functions of the optimal system and represent the optimal control solution in terms of the state and co-state functions. The main goal of this principle (PMP) is to minimize the objective function. Thus depending on the constraints in the objective function, we will minimize the Hamiltonian function, $\mathrm{H}$ with respect to the controls and define the co-state functions as $\lambda_{S}, \lambda_{V}, \lambda_{I}, \lambda_{R}$ and $\lambda_{B}$ associated with the state equations defined for $\mathrm{S}, \mathrm{V}, \mathrm{I}, \mathrm{R}$ and $\mathrm{B}$. We further note that from (21) Theorem 3.1 holds.

Let S, V, I, R and B be the optimal state solutions with associated optimal control variables $u_{1}, u_{2}, u_{3}$ for the optimal control model system (1)-(5), there exist co-state variables $\lambda_{S}, \lambda_{V}, \lambda_{I}, \lambda_{R}$ and $\lambda_{B}$. Thus to achieve the optimal control, our co-state functions must satisfy:

$$
\frac{d \lambda_{S}}{d t}=-\frac{\partial H}{\partial S}, \frac{d \lambda_{V}}{d t}=-\frac{\partial H}{\partial V} \frac{d \lambda_{I}}{d t}=-\frac{\partial H}{\partial I}, \frac{d \lambda_{R}}{d t}=-\frac{\partial H}{\partial R}, \frac{d \lambda_{B}}{d t}=-\frac{\partial H}{\partial B} .
$$


Where

$$
\begin{aligned}
& \frac{\partial H}{\partial S}=L_{1}-\lambda_{S}\left(\beta B(t)+u_{1}+\mu\right)+\lambda_{V} u_{1}+\lambda_{I} \beta B(t) \\
& \frac{\partial H}{\partial V}=\lambda_{S} b-\lambda_{V}(b+\mu) \\
& \frac{\partial H}{\partial I}=L_{2}-\lambda_{1}\left(d+\alpha+\mu+u_{2}\right)+\lambda_{R}\left(\alpha+u_{2}\right)+\lambda_{B} \eta \\
& \frac{\partial H}{\partial R}=-\lambda_{R} \mu \\
& \frac{\partial H}{\partial B}=-\lambda_{S} \beta S(t)+\lambda_{I} \beta S(t)+\lambda_{B}\left(\gamma-\mu_{1}-u_{3}\right)
\end{aligned}
$$

Thus

$$
\begin{aligned}
\frac{d \lambda_{S}}{d t} & =\lambda_{S}\left(\beta B(t)+u_{1}+\mu\right)-L_{1}-\lambda_{V} u_{1}-\lambda_{I} \beta B(t) \\
\frac{d \lambda_{V}}{d t} & =\lambda_{V}(b+\mu)-\lambda_{S} b \\
\frac{d \lambda_{I}}{d t} & =\lambda_{1}\left(d+\alpha+\mu+u_{2}\right)-L_{2}-\lambda_{R}\left(\alpha+u_{2}\right)-\lambda_{B} \eta \\
\frac{d \lambda_{R}}{d t} & =\lambda_{R} \mu \\
\frac{d \lambda_{B}}{d t} & =\lambda_{S} \beta S(t)-\lambda_{I} \beta S(t)-\lambda_{B}\left(\gamma-\mu_{1}-u_{3}\right)
\end{aligned}
$$

With transversality or final time conditions;

$$
\left[\lambda_{S}(T), \lambda_{V}(T), \lambda_{I}(T), \lambda_{R}(T), \lambda_{B}(T)\right]=0 .
$$

The characterization of the optimal controls $u_{1}^{*}(t), u_{2}^{*}(t), u_{3}^{*}(t)$, that is the optimality equations are based on the conditions:

$$
\frac{\partial H}{\partial u_{1}}=\frac{\partial H}{\partial u_{2}}=\frac{\partial H}{\partial u_{3}}=0
$$

for

$$
\begin{aligned}
& \frac{\partial H}{\partial u_{1}}=M_{1} u_{1}-S(t) \lambda_{S}+\lambda_{V} S(t)=0 \\
& \frac{\partial H}{\partial u_{2}}=M_{2} u_{2}-\lambda_{I} I(t)+\lambda_{R} I(t)=0 \\
& \frac{\partial H}{\partial u_{3}}=M_{3} u_{3}-\lambda_{B} B(t)=0
\end{aligned}
$$

Subject to the constraints with $0 \leq u_{1} \leq 1, \quad 0 \leq u_{2} \leq 1, \quad 0 \leq u_{3} \leq 1$ and solving for $u_{1}=u_{1}^{*}, u_{2}=u_{2}^{*}, u_{3}=u_{3}^{*}$ from the above set of equations, the optimality conditions are obtained as follows:

$$
\begin{aligned}
& u_{1}^{*}=\frac{\left(\lambda_{s}-\lambda_{V}\right) S(t)}{M_{1}} \\
& u_{2}^{*}=\frac{\left(\lambda_{I}-\lambda_{R}\right) I(t)}{M_{2}} \\
& u_{3}^{*}=\frac{\lambda_{B} B(t)}{M_{3}}
\end{aligned}
$$

Thus using the bounds of the control its optimal control $u_{1}(t)$ is given by

$$
u_{1}^{*}= \begin{cases}\frac{\left(\lambda_{S}-\lambda_{V}\right) S(t)}{M_{1}} & \text { if } 0 \leq \frac{\left(\lambda_{S}-\lambda_{V}\right) S(t)}{M_{1}} \leq 1 \\ 0 & \text { if } \frac{\left(\lambda_{S}-\lambda_{V}\right) S(t)}{M_{1}} \leq 0 \\ 1 & \text { if } \frac{\left(\lambda_{S}-\lambda_{V}\right) S(t)}{M_{1}} \geq 1\end{cases}
$$


Control $u_{1}(t)$ can be written in compact form as

$$
u_{1}^{*}=\min \left\{\max \left\{0, \frac{\left(\lambda_{s}-\lambda_{V}\right) S(t)}{M_{1}}, 1\right\}\right\}
$$

Also using the bounds of the control $u_{2}(t)$, its optimal control is given by;

$$
u_{2}^{*}= \begin{cases}\frac{\left(\lambda_{1}-\lambda_{R}\right) I(t)}{M_{2}} & \text { if } 0 \leq \frac{\left(\lambda_{1}-\lambda_{R}\right) I(t)}{M_{2}} \leq 1 \\ 0 & \text { if } \frac{\left(\lambda_{I}-\lambda_{R}\right) I(t)}{M_{2}} \leq 0 \\ 1 & \text { if } \frac{\left(\lambda_{I}-\lambda_{R}\right) I(t)}{M_{2}} \geq 0 .\end{cases}
$$

Again control $u_{2}(t)$ can be written in compact form as

$$
u_{2}^{*}=\min \left\{\max \left\{0, \frac{\left(\lambda_{I}-\lambda_{R}\right) I(t)}{M_{2}}, 1\right\}\right\}
$$

Using the bounds of the control $u_{3}(t)$, its optimal control is given by;

$$
U_{3}^{*}= \begin{cases}\frac{\lambda_{B} B(t)}{M_{3}} & \text { if } 0 \leq \frac{\lambda_{B} B(t)}{M_{3}} \leq 1, \\ 0 & \text { if } \frac{\lambda_{B} B(t)}{\lambda_{3}} \leq 0 \\ 1 & \text { if } \frac{\lambda_{B} B(t)}{M_{3}} \geq 0\end{cases}
$$

In compact form, control $u_{3}(t)$ can be written as

$$
u_{3}^{*}=\min \left\{\max \left\{0, \frac{\lambda_{B} B(t)}{M_{3}}, 1\right\}\right\}
$$

From equations (22), (23) and (24) we obtain the following optimality system

$$
\begin{aligned}
& \frac{d S}{d t}=(1-\rho) A-\beta S(t) B(t)+b V(t)-\min \left\{\max \left\{0, \frac{\left(\lambda_{S}-\lambda_{V}\right) S(t)}{M_{1}}, 1\right\}\right\} S(t)-\mu S(t), \\
& \frac{d V}{d t}=\rho A+\min \left\{\max \left\{0, \frac{\left(\lambda_{S}-\lambda_{V}\right) S(t)}{M_{1}}, 1\right\}\right\} S(t)-b V(t)-\mu V(t), \\
& \frac{d I}{d t}=\beta S(t) B(t)-(d+\alpha+\mu) I(t)-\min \left\{\max \left\{0, \frac{\left(\lambda_{I}-\lambda_{R}\right) I(t)}{M_{2}}, 1\right\}\right\} I(t), \\
& \frac{d R}{d t}=\alpha I(t)-\mu R(t)+\min \left\{\max \left\{0, \frac{\left(\lambda_{I}-\lambda_{R}\right) I(t)}{M_{2}}, 1\right\}\right\} I(t),
\end{aligned}
$$




$$
\begin{aligned}
\frac{d B}{d t} & =\gamma B(t)+\eta I(t)-\mu_{1} B(t)-\min \left\{\max \left\{0, \frac{\lambda_{B} B(t)}{M_{3}}, 1\right\}\right\} B(t), \\
\frac{d \lambda_{S}}{d t} & =\lambda_{S}\left(\beta B(t)+\min \left\{\max \left\{0, \frac{\left(\lambda_{S}-\lambda_{V}\right) S(t)}{M_{1}}, 1\right\}\right\}+\mu\right)-L_{1} \\
& -\lambda_{V} \min \left\{\max \left\{0, \frac{\left(\lambda_{S}-\lambda_{V}\right) S(t)}{M_{1}}, 1\right\}\right\}-\lambda_{I} \beta B(t) \\
\frac{d \lambda_{V}}{d t} & =\lambda_{V}(b+\mu)-\lambda_{S} b \\
\frac{d \lambda_{I}}{d t} & =\lambda_{1}\left(d+\alpha+\mu+\min \left\{\max \left\{0, \frac{\left(\lambda_{I}-\lambda_{R}\right) I(t)}{M_{2}}, 1\right\}\right\}\right)-L_{2} \\
& -\lambda_{R}\left(\alpha+\min \left\{\max \left\{0, \frac{\left(\lambda_{I}-\lambda_{R}\right) I(t)}{M_{2}}, 1\right\}\right\}\right)-\lambda_{B} \eta \\
\frac{d \lambda_{R}}{d t} & =\lambda_{R} \mu \\
\frac{d \lambda_{B}}{d t} & =\lambda_{S} \beta S(t)-\lambda_{I} \beta S(t)-\lambda_{B}\left(\gamma-\mu_{1}-\min \left\{\max \left\{0, \frac{\left(\lambda_{S}-\lambda_{V}\right) S(t)}{M_{1}}, 1\right\}\right\}\right)
\end{aligned}
$$

with $S(0)=S_{0}, V(0)=V_{0}, I(0)=I_{0}, R(0)=R_{0}, B(0)=B_{0}$ and

$$
\lambda_{S}(T)=\lambda_{V}(T)=\lambda_{I}(T)=\lambda_{R}(T)=\lambda_{B}(T)=0
$$

We note that the optimality system consists of the state equations with the initial conditions, the co-state equations plus the final time conditions and the optimal control solution. All these are solved numerically in Section 4.

\section{Numerical Simulations}

Under this section, we numerically solve the model system (1)-(5) together with the control systems. We apply the Forward Runge-Kutta fourth order scheme, Devipriya and Kalaivani (2012) to compute the optimality control solution as well as the transversality conditions given in (25). The state equations are solved using the initial values given in Table 3 , for the different simulations. Various constant parameters used in the objective function are; $L_{1}=0.02, L_{2}=10, M_{1}=$ $10, M_{2}=10, M_{3}=20$. The parameter values used in the simulation are stated in Table 3 with the set initial conditions and we consider the entire period $T=100$ days. 
Table 3. State variables and parameters of the Model with control strategies

\begin{tabular}{|c|c|c|c|}
\hline Variables & Description & Value & Reference \\
\hline$S(0)$ & $\begin{array}{l}\text { Number of the susceptible at } \\
\text { time } t=0\end{array}$ & 100 & Assumed \\
\hline$V(0)$ & $\begin{array}{l}\text { Number of the vaccinated at } \\
\text { time } t=0\end{array}$ & 25 & Assumed \\
\hline$I(0)$ & $\begin{array}{l}\text { Number of infected at time } \\
t=0\end{array}$ & 50 & Assumed \\
\hline$R(0)$ & $\begin{array}{l}\text { Number of recovered at time } \\
t=0\end{array}$ & 10 & Assumed \\
\hline$B(0)$ & $\begin{array}{l}\text { Free pathogen environmen- } \\
\mathrm{t} \text { at time } t=0\end{array}$ & 100 & Assumed \\
\hline \multicolumn{4}{|l|}{ Parameters } \\
\hline$A$ & Recruitment rate & 10 per day & Assumed \\
\hline$\beta$ & $\begin{array}{l}\text { Contact rate to transmit the } \\
\text { disease }\end{array}$ & $\begin{array}{l}0.2143 \text { per } \\
\text { day }\end{array}$ & {$[22]$} \\
\hline$\rho$ & $\begin{array}{l}\text { Proportion of infants with } \\
\text { maternal immunity }\end{array}$ & 0.6 & {$[15]$} \\
\hline$\rho$ & Proportion of vaccination & 0.85 & Assumed \\
\hline$\alpha$ & Recovery rate & 0.2 per day & {$[15,23]$} \\
\hline$b$ & $\begin{array}{l}\text { Rate at which the vaccine } \\
\text { wears off }\end{array}$ & 0.005 per day & Assumed \\
\hline$d$ & Disease induced death rate & 0.015 & {$[23]$} \\
\hline$\eta$ & $\begin{array}{l}\text { Contribution of the infected } \\
\text { to environment }\end{array}$ & $\begin{array}{l}10 \text { cell/L per } \\
\text { day }\end{array}$ & Assumed \\
\hline$\gamma$ & $\begin{array}{l}\text { Generation of the pathogen } \\
\text { population }\end{array}$ & 0.073 per day & Assumed \\
\hline$\mu$ & Natural death rate of human & $\begin{array}{l}0.0000548 \\
\text { per day }\end{array}$ & {$[23]$} \\
\hline$\mu_{1}$ & $\begin{array}{l}\text { Rate of death of the cholera } \\
\text { disease }\end{array}$ & 1.06 per day & {$[23]$} \\
\hline
\end{tabular}

\subsection{Optimal Vaccination Only}

Under this strategy, we use the control $u_{1}$ to optimize the objective function while $u_{2}$ and $u_{3}$ are set to zero. From Figure 2(a) control $u_{1}$ is maximum from $t=0$ to $t=64$ days and drops rapidly to zero. This implies that vaccination is effective from the beginning and should be fully implemented. When there is vaccination, the susceptible individuals take longer to leave the susceptible class because their degree of susceptibility is low than when there is no vaccination. Furthermore infected individuals take less time to recover in the presence of vaccination ( 7 days) compared to when there is no vaccination (15 days) as shown in Figure 2(b)

\subsection{Optimal Health Education Campaigns Only}

Under this strategy we use control $u_{3}$ to optimize the objective function while $u_{1}$ and $u_{2}$ is set to zero. From Figure 3(a) controls $u_{3}$ is maximum from $t=0$ to $t=74$ days and drops rapidly to zero. This implies that education campaigns are effective from the beginning and should be fully implemented like in the case of vaccination. In 3(c) it is clearly shown that the control reduces on the number of the free pathogen to 400 cell/ $\mathrm{L}$ as compared to $1800 \mathrm{cell} / \mathrm{L}$ when there is no health awareness and it greatly reduces the pathogen existence. We further note that in figure $3(\mathrm{~b})$ health education campaigns have no effect on the vaccinated class. 


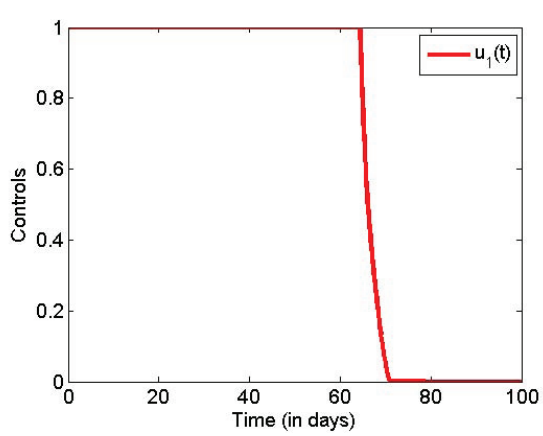

(a) $u_{1}$ control

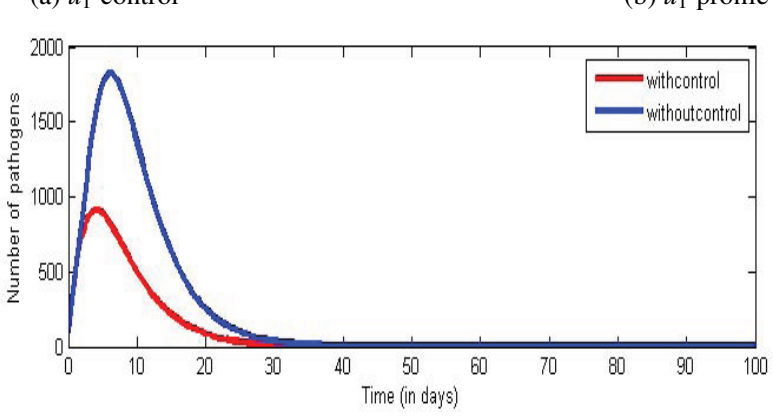

(c) $u_{1}$ profile on environment
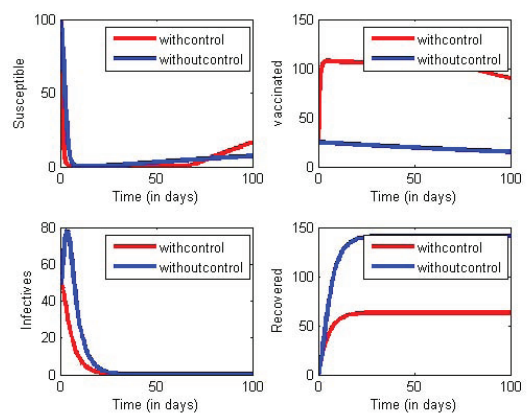

(b) $u_{1}$ profile

Figure 2. A curve showing the influence of vaccination on the dynamics of cholera disease

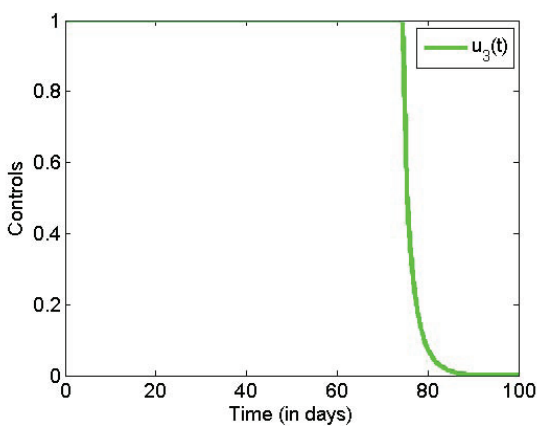

(a) $u_{3}$ control
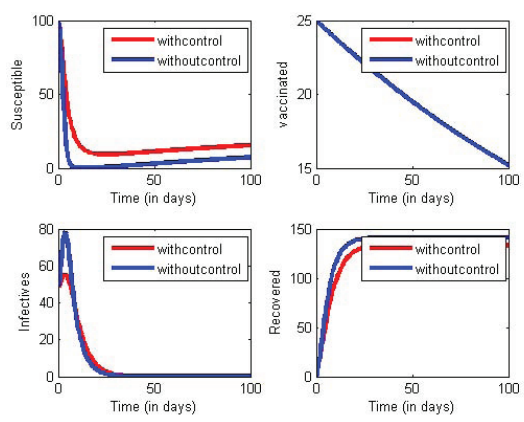

(b) $u_{3}$ profile

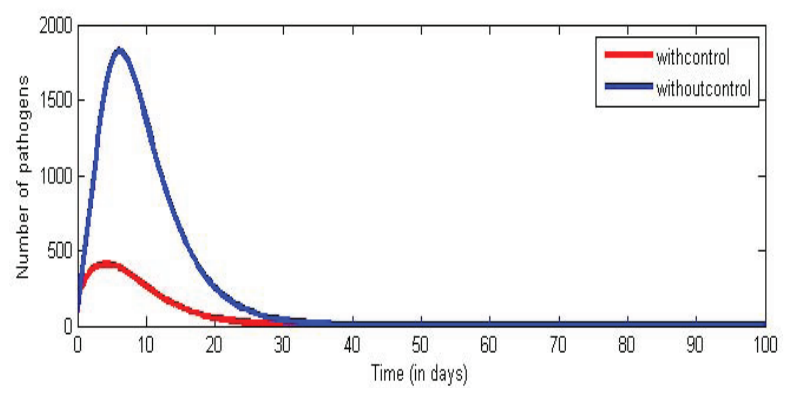

(c) $u_{3}$ profile on pathogen environment

Figure 3. A curve showing the influence of health education campaigns on cholera disease

\subsection{Optimal Vaccination and Health Campaigns Only}

Under this strategy we use two controls $u_{1}$ and $u_{3}$ to optimize the objective function while $u_{2}$ is set to zero. 


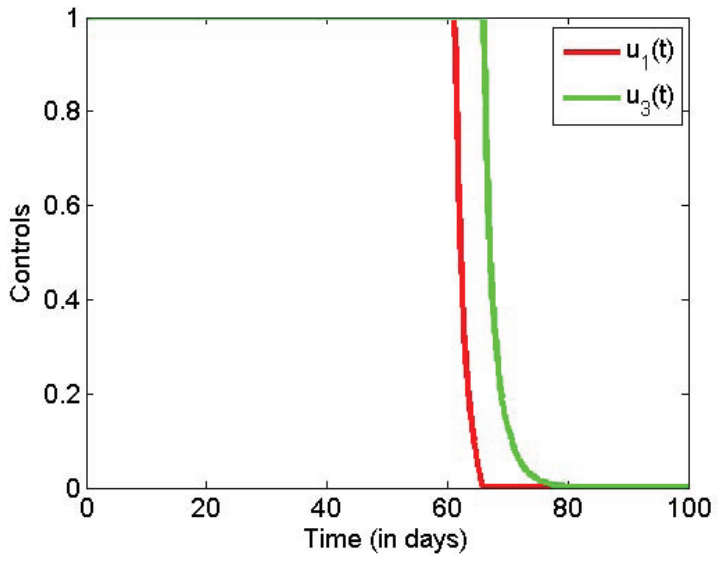

(a) $u_{1}$ and $u_{3}$ controls
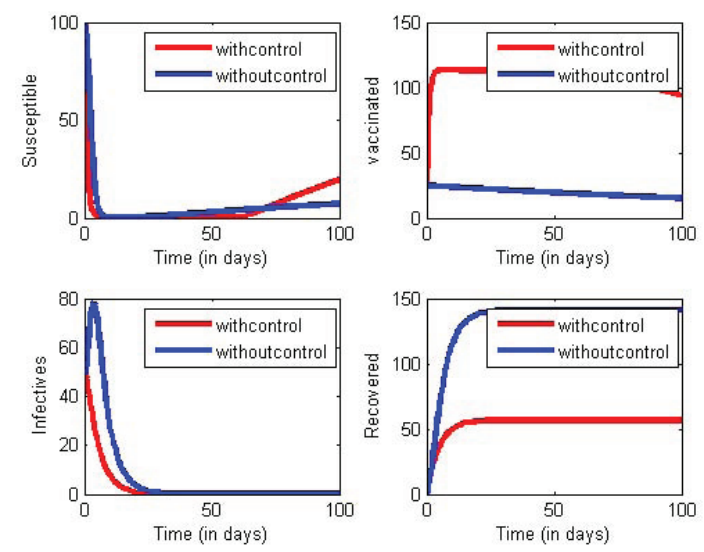

(b) $u_{1}$ and $u_{3}$ profiles

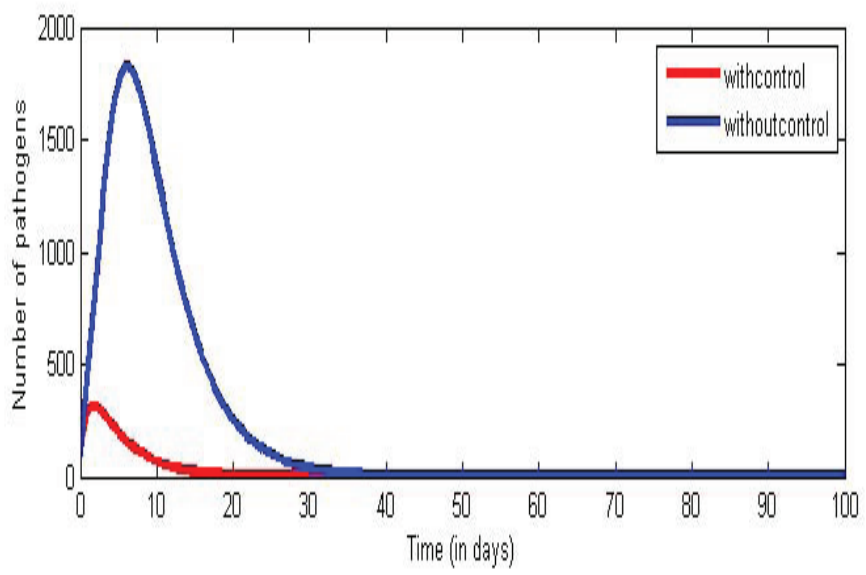

(c) $u_{1}$ and $u_{3}$ profile on pathogen environment

Figure 4. A curve showing the influence of vaccination and health education campaigns on cholera disease

Figure 4(a) shows that $u_{1}$ is maximum from $t=0$ to $t=61$ days and the control is maximum from $t=0$ to $t=66$ days and they both gradually decrease to zero, this implies that vaccination and education campaigns should be administered fully from the start. With these two controls applied, the disease will disappear within the first 15 days as shown in Figure 4(b) and with both these controls in place the pathogen population will reduce from 1800 cell/L to 290 cell/L as shown in Figure 4(c) hence these two controls are effective in eliminating cholera disease.

\subsection{Optimal Vaccination, Treatment and Health Education Campaigns}

Under this strategy, we use all controls, that is, $u_{1}, u_{2}$ and $u_{3}$ to optimize the objective function. In Figure 5 (a), $u_{1}$ and $u_{3}$ are maximum from $t=0$ to $t=61$ and $t=0$ to $t=66$ days respectively and drop rapidly to zero. $u_{2}$ is low throughout the time. This implies that vaccination and education campaigns should be applied right away from the beginning. Treatment is low because if more people are vaccinated then a few will get sick. With all the controls applied the disease will disappear within 18 days as shown in Figure 5(b). With no controls the disease will persist for about 22 days and the pathogens for about 16 days as shown in Figure 5(c).

\section{Conclusion}

The main purpose of this work was to formulate and analyze a mathematical model of the dynamics and optimal control strategies of cholera disease. We have presented and analyzed a cholera model with controls, $u_{1}$ for vaccination of the human population, $u_{2}$ for treatment and $u_{3}$ for health education campaigns. We derived and analyzed the conditions for optimal control of the cholera disease using the Pontryagin's maximum principle, and simulated for different control strategies. The results show that vaccination and education campaigns should be applied from the start followed by treatment. The purpose of education campaigns were to let individuals understand the risks in the contaminated environment, 


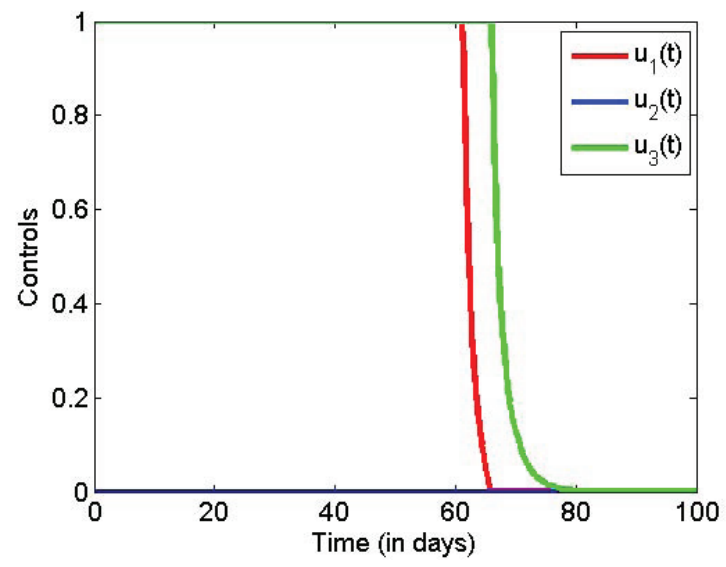

(a) $u_{1}, u_{2}$ and $u_{3}$ controls
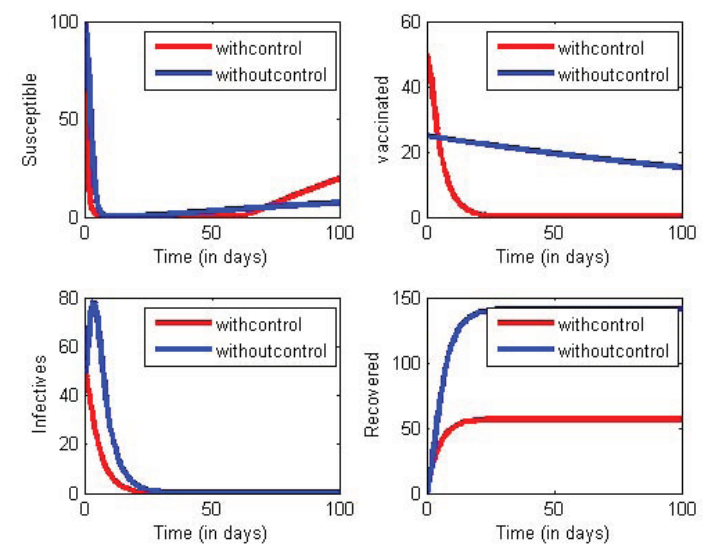

(b) $u_{1}, u_{2}$ and $u_{3}$ profiles

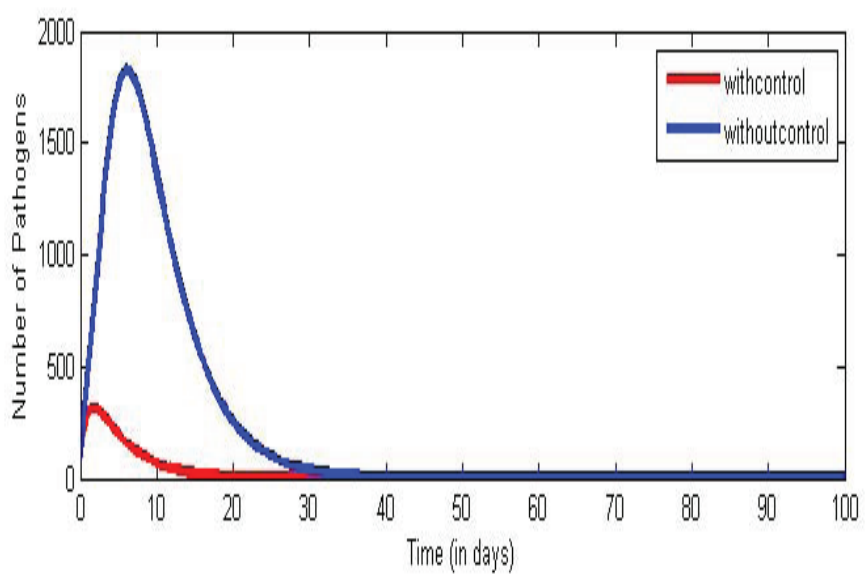

(c) $u_{1}, u_{2}$ and $u_{3}$ profile on pathogen environment

Figure 5. A curve showing the influence of vaccination, treatment and health education campaigns on cholera disease

the need of vaccination was to ensure that the degree of susceptibility is as low as possible and treatment was to reduce the number of infected individuals.

\section{Conflict of Interests}

The authors declare that there is no conflict of interests.

\section{References}

Al-Arydah, M. T., Mwasa, A., Tchuenche, J. M., \& SMITH, R. J. (2013). Modeling Cholera Disease with Education and Chlorination. Journal of Biological Systems, 21(04), 1340007.

Ali, M., Lopez, A. L., You, Y., Kim, Y. E., Sah, B., Maskery, B., \& Clemens, J. (2012).The global burden of cholera. Bulletin of the World Health Organization, 90(3), 209-218.

Bartlett, A. (1991). Cholera lessons. The Lancet, 338(8776), 1216.

Barzilay, E. J., Schaad, N., Magloire, R., Mung, K. S., Boncy, J., Dahourou, G. A., \& Tappero, J. W. (2013). Cholera surveillance during the Haiti epidemicthe first 2 years. New England, Journal of Medicine, 368(7), 599-609.

Bashiru, I., \& Asokan, C. (2016). Quantification of Escherichia Coli Concentration in Water in Some Selected Households in Namanyonyi Sub-County, Mbale District, Uganda. The International Journal of Science and Technology, 4(2), 121.

Birkhorff. G, \& Rota,G. C. (1982). Ordinary Differential Equations. Needham Heights, Ginn.

Biswas, D. K., Bhunia, R., Maji, D., \& Das, P. (2014). Contaminated Pond Water Favors Cholera Outbreak at Haibatpur 
Village, Purba Medinipur District, West Bengal, India. Journal of tropical medicine, 2014.

Chao, D. L., Halloran, M. E., \& Longini, I. M. (2011).Vaccination strategies for epidemic cholera in Haiti with implications for the developing world. Proceedings of the National Academy of Sciences, 108(17), 7081-7085.

Dannan, F. M., \& Elaydi, S. (1986). Lipschitz stability of nonlinear systems of differential equations. Journal of mathematical analysis and applications, 113(2), 562-577.

Devipriya, G., \& Kalaivani, K. (2012). Optimal Control of Multiple Transmission of Water-Borne Diseases. International Journal of Mathematics and Mathematical Sciences, 2012.

Diekmann, O., Heesterbeek J. A., \& Metz J. A. J. (1990). On the Definition and the Computation of the Basic Reproductive Ratio, $R_{0}$ in Models of Infectious Diseases in Heterogeneous Populations, J. Math. Biol., 28, $365-382$.

Gleick, P. H., \& Ajami, N. (2014).The World's Water Volume 8: The Biennial Report on Freshwater Resources (Vol. 8). Island press.

Grad, Y. H., Miller, J. C., \& Lipsitch, M. (2012). Cholera modeling: challenges to quantitative analysis and predicting the impact of interventions. Epidemiology (Cambridge, Mass.), 23(4), 523.

Isere, A. O., Osemwenkhae, J. E., \& Okuonghae, D. (2014).Optimal control model for the outbreak of cholera in Nigeria.African Journal of Mathematics and Computer Science Research, 7(2), 24-30.

Johnson, T., \& McQuarrie, B. (2009). Mathematical Modeling of Diseases: Susceptible-Infected-Recovered (SIR) Model. In University of Minnesota, Morris, Math 4901 Senior Seminar.

Miller£ N. R. L. (2009). Optimal Control Applied to Population and Disease Models. Doctoral Dissertations, 74.

Namawejje, H., Luboobi, L. S., Kuznetsov, D., \& Wobudeya, E. (2014). Modeling optimal control of rotavirus disease with different control strategies. Journal of Mathematical and Computational Science, 4(5), 892.

Neilan, R. L. M., Schaefer, E., Gaff, H., Fister, K. R., \& Lenhart, S. (2010). Modeling optimal intervention strategies for cholera. Bulletin of mathematical biology, 72(8), 2004-2018.

Ochoche, J. M. (2013). A Mathematical Model for the Transmission Dynamics of Cholera with Control Strategy. International Journal of Science and Technology, 2(11).

Pontryagin, L. S. (1962). V. G. Boltyanskii, R. V. Gamkrelidze, \& E. F. Mischenko, Mathematical Theory of Optimal Processes, Wiley, New York.

Ugwa, K. A., Agwu, I. A., \& Agbanyim, A. N. (2013). Mathematical Analysis Of The Endemic Equilibrium Of The transmission Dynamics Of Tuberculosis. International Journal of Technology Enhancements and Emerging Engineering Research, 2(12), 263-269.

Van den Driessche, P., \& Watmough, J. (2002). Reproduction numbers and sub-threshold endemic equilibria for compartmental models of disease transmission. Mathematical biosciences, 180(1), 29-48.

Vogt, A. P., Doshi, R. K., Higgins, J. E., Burd, E. M., Ribner, B. S., \& Kraft, C. S. (2010). Acute cholecystitis caused by nontoxigenic Vibrio cholerae O1 Inaba. Journal of clinical microbiology, 48(3), 1002-1004.

Wang, J., \& Modnak, C. (2011).Modeling cholera dynamics with controls. Canadian Applied Mathematics Quarterly, $19(3), 255-273$.

World Health Organization. (1993). Guidelines for cholera control.

Zhou, X., \& Cui, J. (2011). Modeling and stability analysis for a cholera model with vaccination. Mathematical Methods in the Applied Sciences, 34(14), 1711-1724

\section{Copyrights}

Copyright for this article is retained by the author(s), with first publication rights granted to the journal.

This is an open-access article distributed under the terms and conditions of the Creative Commons Attribution license (http://creativecommons.org/licenses/by/4.0/). 